\title{
New antibiotic hits Gram-negative bacteria
}

The spread of multidrug-resistant (MDR) bacteria is a serious public health threat, and new drug options for Gram-negative bacteria are in particularly urgent need. A group of researchers at Genentech have developed a new antibiotic derived from arylomycins that is effective against MDR clinical isolates of the most relevant Gram-negative pathogenic species and shows low potential for development of resistance.

Gram-negative bacteria possess a dual membrane envelope, which makes them particularly challenging to target. Indeed, no new classes of antibiotics against such pathogens have been developed in over 50 years. The situation is particularly worrisome for a group of Gram-negative bacteria found predominantly in hospitals. Without an entirely new way to combat them, Gram-negative bacteria pose a serious threat to a

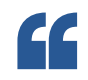

Without an

entirely new

way to

combat them, Gram-negative bacteria pose

\section{a serious}

threat to

a highly

vulnerable

population

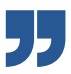

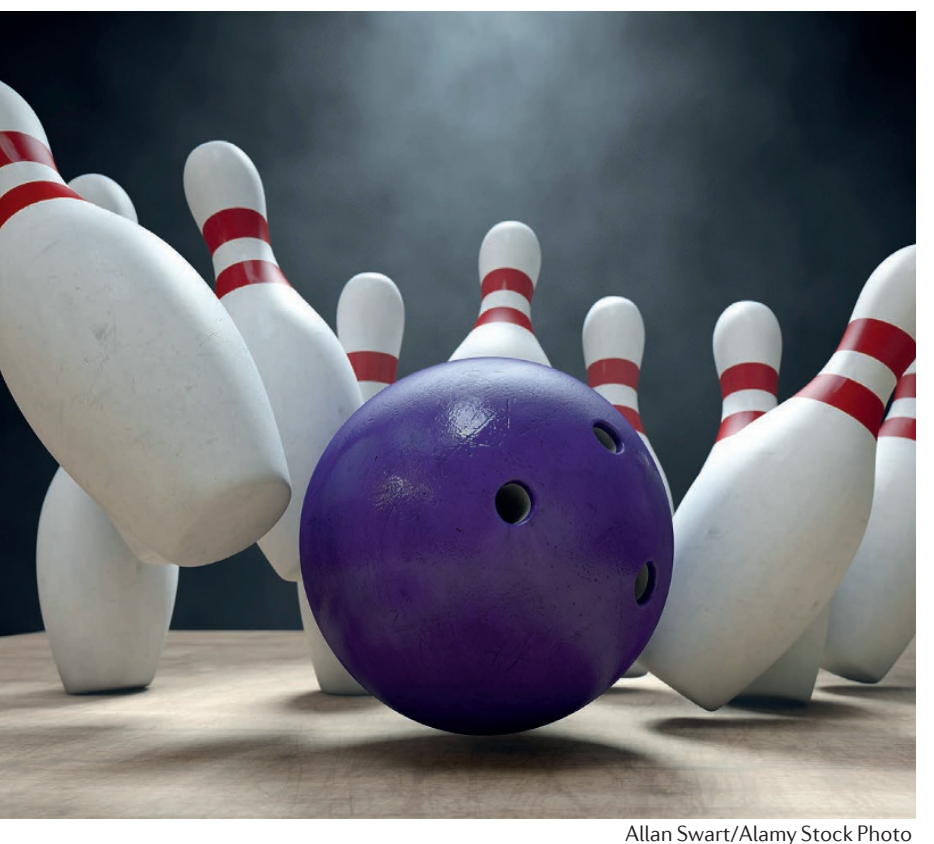

highly vulnerable population, note Christopher Heise and Peter Smith, who both led the study.

For the current study, Heise and colleagues focused on modifying arylomycins, a class of macrocyclic lipopeptides that inhibit an essential bacterial protease called type I signal peptidase (SPase). In Gram-negative bacteria, the active site of SPase lies in the periplasmic space between the dual membranes. The researchers wanted to modify arylomycins to better access this space and improve the target binding affinity.

The researchers left the macrocyclic core of the arylomycin scaffold unchanged, as crystallographic data showed that this domain occupies a conserved region of the substrate-binding pocket of the target. Modifications to other regions of the molecule included addition of electrophilic warheads to the C-terminal domain, with the aim of promoting covalent binding to the catalytic domain of SPase.

The resultant molecule, termed G0775, was able to penetrate the bacterial outer membrane and showed potent in vitro antibacterial activity against a panel of MDR Gram-negative clinical isolates, including strains of Escherichia coli and Pseudomonas aeruginosa. Notably, G0775 was active against a strain of Klebsiella pneumoniae that was resistant to 13 existing classes of antibiotics.

At low G0775 concentrations, resistance among various Gramnegative pathogens arose at a moderate rate, as expected for a drug that targets a single gene product. However, increasing the concentration of G0775 to eight times the minimal inhibitory concentration (MIC) reduced the frequency of resistance below the limit of detection for K. pneumoniae and Acinetobacter baumannii. A concentration of 16 times the MIC cut the rate of resistance to less than $10^{-10}$ in E. coli and P. aeruginosa.

To probe the molecular mechanism of G0775 activity, Heise and colleagues examined co-crystal structures of the compound bound to SPase. They observed an intriguing mode of drug binding in which the electrophilic warhead moieties of G0775 formed a covalent amidine through a lysine residue on the SPase catalytic site.

To test the in vivo efficacy of G0775, the researchers first used a thigh infection model in neutropenic mice. The drug had potent bactericidal activity against strains of $E$. coli and K. pneumoniae, and at higher concentrations against $P$. aeruginosa and A. baumannii. Moreover, G0775 had dose-dependent antibacterial activity against MDR K. pneumoniae in a mouse model of pulmonary infection, and increased survival in a peritonitis model of K. pneumoniae infection.

Importantly, the unique design of G0775 and mechanism of interaction with SPase allows this molecule to circumvent known mechanisms of resistance that typically manifest in Gram-negative bacteria, say Heise and Smith.

These studies highlight optimized arylomycins as a promising new class of antibiotics that could be used to combat a broad spectrum of drugresistant Gram-negative bacteria.

Katie Kingwell 